\title{
Analysis and Modeling of Time Series Based on Granular Computing
}

\author{
Min Tu, Yuanjian Zhang, Jianfeng Xu, and Yu Li
}

\begin{abstract}
Granular computing is an important theory in solving complicated problems. Currently, researches of granular computing on time series stress more on the design of algorithms, the modeling of time series using information granular is limited. This paper proposed a granular-based model called Temporal Information System (TIS) on time series by using equivalent relationship, which is an extension of granular computing. Major works in modeling include the granulation of time series, development of granular layer as well as the transition rules. The granular operation and its properties provide a novel method for further research on time series which are complicated and uncertain in real applications.
\end{abstract}

Index Terms-Granular computing, time series, temporal information system, equivalent relationship, granular operation.

\section{INTRODUCTION}

Humans are accustomed to analyze the structure of discourse from different perspectives by extracting the essence and mapping to different levels and reorganize the structure according to the nature and characteristics of the problem domain. It is more obvious when tackling with the complex problem with the properties of uncertainty, ambiguity, and high dimension to get the satisfied result. Although there are many ways to solve complex problem, these analyzes have established a common reasoning characteristic of ordered structures at different levels. This kind of thinking reflects the idea of granular computing. Quotient space [1] and rough sets [2] are classical models of granular computing.

Time series is a commonly researched data reflecting the state transition of certain objects by ordered observations [3]. There are many applications that generate the temporal data, such as monitoring and forecasting the lightning according to meteorological factors, monitoring the patient physiological in order to rescue in time, indoor environmental monitoring to conserve electrical energy consumption. These applications are usually composed of one or a plurality of attributes, and the complexity of internal relationship is much higher than the conventional information system [4] denoted as $\mathrm{IS}=(\mathrm{U}, \mathrm{A}, \mathrm{V}, \mathrm{f})$. How to describe the relationship under the background of time series so that the knowledge is

Manuscript received September 3, 2014; revised December 19, 2014 This work was supported in part by Chinese National Natural Science Fund (No. 61070139).

Min Tu is with the Jiangxi Police College, Nanchang, 330100, China (e-mail: tumin_y@163.com).

Yuanjian Zhang, Jianfeng $\mathrm{Xu}$, and $\mathrm{Yu} \mathrm{Li}$ are with Nanchang University, Nanchang, 330047, China (e-mail: zhangyj901029@gmail.com, jianfeng.nc@gmail.com, ly4232@gmail.com). well-structured is an important issue.

Currently the research on time series is mainly conducted from a methodological point of view, the main method can be divided into two categories, namely time series segments (use the window as its basic semantics) [5]-[8] based and overall characteristics based [9]. Although these methods have achieved good results in applications, these design of algorithms and selection of parameters are often closely related to the specific application areas. In the era of big data, the portable of traditional designs are limited, making time series data modeling and reasoning an urgent issue. Granular computing domain in practice can not only granulating the discourse, but the granular can be calculated a wealth of relationships such as thickness and sequence. This kind of decomposition can preserve the semantic well. The existing methods on dealing with time series can be unified to the granular computing ideology due to the scope of processing object can be explained as the difference of thickness level and different structure and morphology is reflected.

In this paper, the basic concepts of granular computing is redefined in the context of time series, while the selection of particle size and morphology is viewed as an important factor in the selection of time series processing .By analyzing the relationship between the level of the time series, the conversion under the rules of different relationships are deduced so that multi-granular analysis on time series can be performed systematically. Granular computing system under time series not only provide theoretical basis for solving complex time series issues, but also enrich and develop the applications of granular computing.

\section{RELATED WORK}

$\mathrm{Xu}$ [6] et al. analyzed the homogenous evolution of information system by using the rough set theory on multi-granular time series. In this paper, the stable of decision rule within a range of time is critically considered. By defining technical terms such as inheriting degree of attribute and inheriting degree of attribute value, the core set of condition in next granular can be deduced. Hu [10] et al. promoted this research and considered the conflict phenomenon between forecasted and actual decision rule. A pay off matrix is established by game theory to analyze the steady of orientation evolution, which provides a novel idea for abnormal detection. The research of both $\mathrm{Xu}$ and $\mathrm{Hu}$ can be viewed as one vertical slice in Fig. 1 (a), as is exemplified in Fig. 1(b) the conversion of time granularity and its impact on decision rule is not considered.

Zuo [11] et al. analyzed the representation of temporal by using interval algebra. By defining instants with granularities 
and intervals with granularities and analyzing the mutual transition rules, the relations of different temporal and their linguistic can be measured and their order in time axis can be computed. The complexity and dynamics of temporal relation are shown in different granular layer. In the subsequent research [12], the temporal granularity is vectorized into different unit of granularity and the operating mechanism among different granularity is derived.

Witold [7] et al. proposed a granular-based and hierarchical model to analyze the stock trade. In this paper, the temporal relation is developed by performing on Cartesian on amplitude and change of amplitude. The adjacent temporal granularity derived in the time series have prominent differences and the semantic is assigned by clustering labels. The form of time series is thus converted to a series of state. Wei Lu [13] et al. proposed a method of developing fuzzy temporal granularity on stock trade. In this research, the semantic of each temporal granular may correspond to one or more fuzzy concept. The hierarchical in both research is shown by multi granular step, the transition of different granularity is not fully considered.

Rami [8] et al. proposed a granular representation framework and use Particle Swarm Optimization (PSO) to optimize the representation of information granular on time series. In this paper, the representation of granular is composed of granular description, granular pattern and granular parameter, making the knowledge of time series more structured. The optimization of information granular is to revise the boundary of granular which is derived by PAA. The certainty of granular is thus become a problem of parameterization.

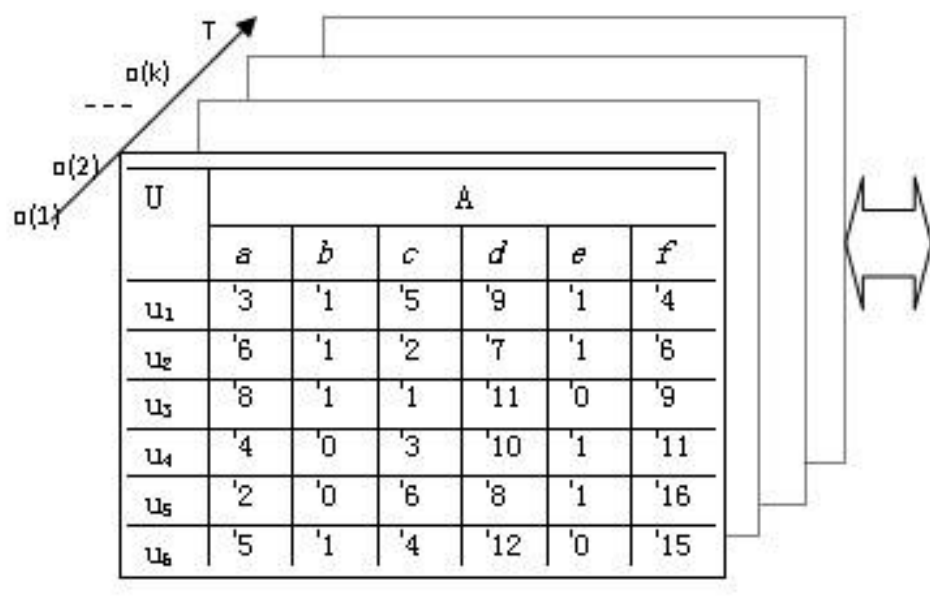

Fig. 1(a). Multi-dimension view of serialized IS.

\begin{tabular}{|c|c|c|c|c|c|c|}
\hline \multirow[t]{2}{*}{$\mathrm{U}$} & \multicolumn{6}{|c|}{ A } \\
\hline & 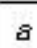 & $b$ & $c$ & $d$ & $e$ & $f$ \\
\hline $\mathrm{u}_{1}$ & ' 3 & '1 & '5 & '9 & 1 & '4 \\
\hline $\mathrm{ul}_{2}$ & 6 & '1 & ' 2 & 'T & 1 & '6 \\
\hline$u_{s}$ & ' 8 & 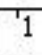 & 1 & 11 & ' & ' 9 \\
\hline $\mathrm{u}_{4}$ & 4 & '0 & 3 & 10 & 1 & 11 \\
\hline$u_{5}$ & ' 2 & '0 & '6 & '8 & 1 & '16 \\
\hline$u_{6}$ & '5 & '1 & 4 & '12 & '0 & '15 \\
\hline
\end{tabular}

Fig. 1(b). View of classical information system.

\section{Granular Model on Time Series}

\section{A. Preliminary Definition}

There are two major aspects in applying granular computing theory, namely how to form information granular and how to reason with the granular. In classical granular computing model such as quotient space and rough set, there are some fundamental terms such as granular, granular relation and the cardicardal of granular. In the discourse of time series, the basic concept of granular computing is redefined after giving some intuitive examples.

Fig. 1(b) showed an information system IS which contain the values of different values on different objects in the discourse U. The granular are $\left\{u_{1}, u_{2},\right\},\left\{u_{4}, u_{5}\right\}\left\{u_{3}, u_{6}\right\}$ if the equivalent relationship act on the subset of attribute $\{b, e\}$ since they denote $\{1,1\},\{0,1\}$ and $\{1,0\}$ respectively. However, the situation will be much more complicated if the axis of time is considered, as is the case shown in Fig. 1(a). We hold the hypothesis that the time axis can be viewed as a collection of ordered dot called atomic time unit and it is the minimum measurement of time. It showed that there are a wealth of ordered IS occur in every $o(i)$, where $i=1,2, \ldots$.

Fig. 2 showed the process of converting serialized IS to a typical TS by projecting one vertical slice in Fig. 1(a) and selecting one typical series ui under the domain of attribute a. By this conversion we want to prove that granular computing theory has the natural relationship with time series. It is

apparent that such information system is different from the tradition discourse discussed in IS and some preliminary terms are given so that a new information system can be developed.

Definition 1: (Atomic Time Unit) Atomic Time Unit is defined as the minimum unit of time with a fixed size which is independent of any real applications. The time axis is thus considered to be ordered numerous atomic time units. The atomic time unit meet the condition $o(i)<o(j)$ when $i<j$. The symbol < denotes the $o(i)$ occurs before $o(j)$.

Definition 2: (Temporal Object) Temporal Object is defined as a fraction of time in a time series denoted by $U_{t}$ and the cardicardal can be measured by Atomic Time Unit, i.e. $\operatorname{card}\left|U_{t}\right|=k^{*} \operatorname{card}|o(i)|$, where $k=1,2, \ldots$.

Definition 3: (Temporal Granular) Temporal Granular is defined as a collection of atomic time unit in a temporal relation.

Definition 4: (Temporal Relation) Temporal Relation is defined as a partitioned representation of Temporal Object with a number of ordered Temporal Granular.

\section{B. Temporal Information System}

Based on Definition 1 to Definition 4, a novel information system called TIS is defined in Definition 5.

Definition 5: (Temporal Information System) Temporal Information System is defined as a quadruple TIS $=\left(U_{t}, A_{R}, R\right.$, 
f), where $U_{t}$ is the discourse which represents a given temporal object, $A_{R}$ is the temporal attribute with all the possible temporal relation $R$, where $R=R_{l}, R_{2}, \ldots$, $\mathrm{f}$ is a mapping function from $A_{R}$ to $R$. For the information of object $u_{i}$ on attribute $a$ in original time series, a corresponding view of TIS is shown in Fig. 3.
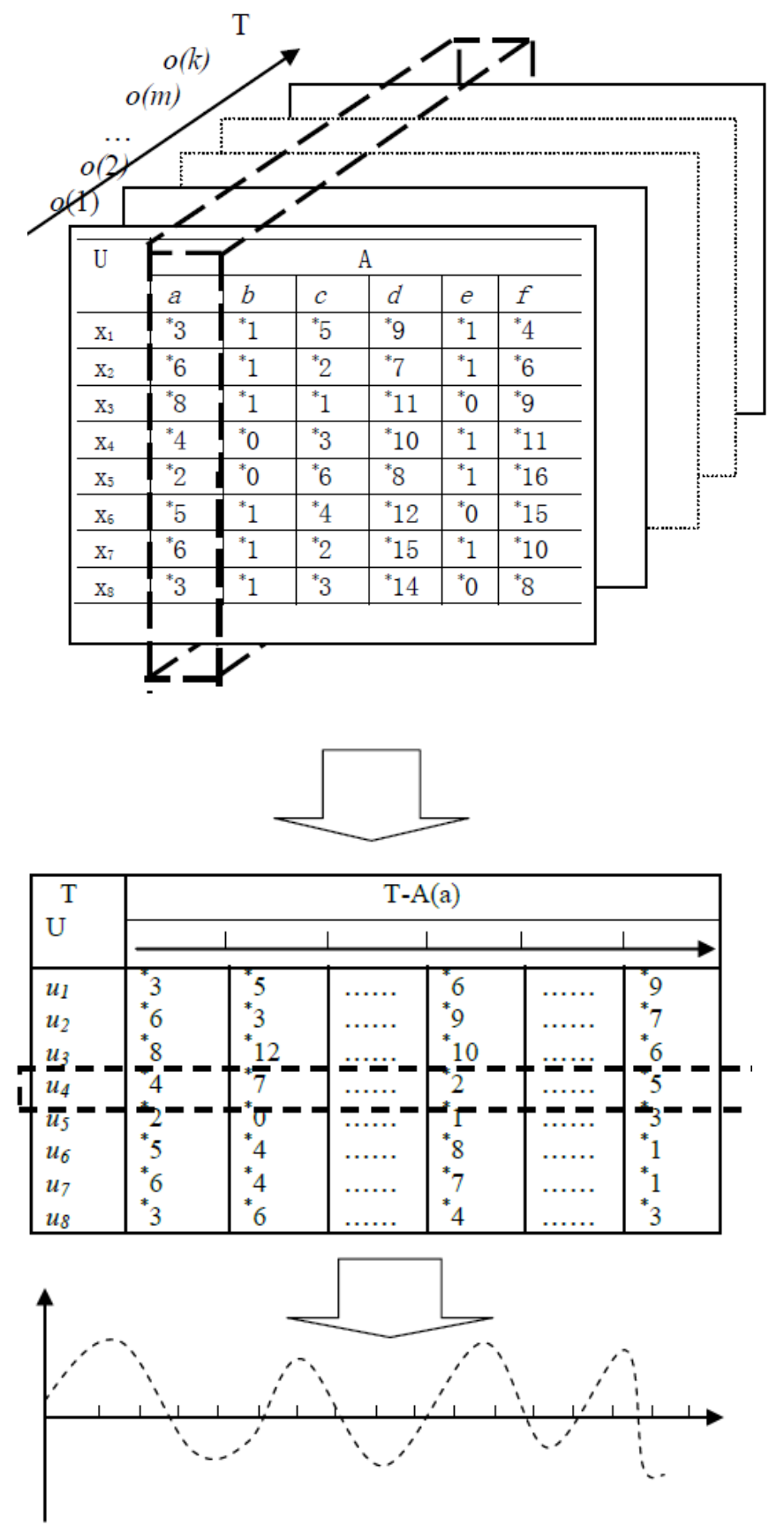

Fig. 2. Conversion from serialized IS to a typical TS.

\begin{tabular}{|c|c|c|c|c|c|}
\hline$R U_{t}^{U_{t}}$ & \multicolumn{5}{|c|}{$\begin{array}{c}U_{t}(i)-A_{R}(a) \\
\mathrm{T} \\
\end{array}$} \\
\hline$R I$ & ${ }^{*} a_{R I(1)}$ & $a_{R l(2)}$ & $a_{R 1(k)}$ & $\ldots \ldots$ & $a_{R 1(m)}$ \\
\hline$R 2$ & $a_{R 2(1)}$ & ${ }^{*} a_{R 2(2)}$ & $a_{R 2(3)}$ & $\ldots \ldots$ & ${ }^{*} a_{R 2(k)}$ \\
\hline R3 & \multicolumn{2}{|c|}{$a_{R 3(1)}$} & $a_{R 3(2)}$ & \multicolumn{2}{|c|}{$\ldots \ldots$} \\
\hline$\ldots \ldots$ & \\
\hline
\end{tabular}

Fig. 3. Example of different temporal relations for a certain attribute of certain object: $(U(i)-A(a))$.

From the perspective of granular, different temporal relation $\mathrm{R}$ can be regarded as different view to restructure a given time objects. For a given temporal relation $R_{i}$, it is composed of a collection of granular whereas each granular is composed of a collection of atomic time unit. By observing the given time objects from different temporal relation, it is possible to have a comprehensive knowledge to analyze the inherent features.

\section{Difference between IS and TIS in Terms of Information Granular}

The way to deduce temporal relation can come from many relation including equivalent relationship, function similar relationship and similar relationship. This paper focus on equivalent relationship and the temporal relation thus is a division on time discourse. Compared with equivalent relationship on traditional granular computing, there are several differences need to be stressed.

1) The recording in granular $* a_{R i(j)}$ have the order relation in time discourse which means their sequence cannot be exchanged, while the granular in traditional granular computing is based on static attribute and thus the $\{a, b\}$ and $\{b, a\}$ have the same meaning.

2) The semantic between granular $* a_{R i(i)}$ and granular $* a_{R i(k)}$ $(j \neq k)$ have the same range denoted by $V_{a}$ since both of them are the measurement of certain attributes in certain objects, whereas the semantic between granular $\{a, b\}$ and $\{b, c\}$ typically do not share the same ranges since they focus on different subset of attributes.

\section{REASONING ON TIS}

\section{A. Granular Operator}

It is observed from Section III that for a given time objects there are a number of temporal relation existed. In order to explain the connections among temporal relations more clearly, some basic granular operators are defined as follows:

\section{1) Unary operator}

Definition 6: (Coarser Operator $(\triangle)$ ) Suppose $* a_{R(i)}$ is a temporal granular defined in temporal relation $\mathrm{R}$ for a TIS, performing coarser operator on $* a_{R(i)}$ is equivalent to coarsing relation $\mathrm{R}$ to be relation $\mathrm{S}$ so that a series of new corresponding granular $* a_{S(j)}$ is derived and meets the condition $* a_{R(i)} \subseteq * a_{S(j)}$ and the relation between temporal relation $R$ and $S$ is denoted as $R \geq S$. It is defined as strict coarser if $\forall * a_{R(i)},{ }^{*} a_{R(i)} \varsubsetneqq * a_{S(j)}$ and the relation between $\mathrm{R}$ and $\mathrm{S}$ is denoted as $R>S$.

The effect of performing coarser operator on original temporal relation can be viewed as merging the granular $* a_{R(i)}$ and $* a_{R(j)}$ and either one condition listed must be satisfied to preserve the order property of granular in new temporal relations:

1) $* a_{R(i)}$ and $* a_{R(j)}$ are consecutive granular in temporal relation $\mathrm{R}$

2) $\forall * a_{R(k)}$ which meet the condition $* a_{R(i)}<* a_{R(k)}<* a_{R(j)}$ or $* a_{R(i)}>* \mathrm{a}_{R(k)}>* a_{R(j)}$ are coarsed meanwhile;

Definition 7: (Refine Operator ( $\left(\right.$ ) ) Suppose $* a_{R(i)}$ is a temporal granular defined in temporal relation $R$ for a TIS, performing refine operator on $* a_{R(i)}$ is equivalent to coarsing 
relation $R$ to be relation $S$ so that a series of new corresponding granular $* a_{S(j)}$ is derived and meets the condition $* a_{S(j)} \subseteq * a_{R(i)}$ and the relation between temporal relation $R$ and $S$ is denoted as $S \geq R$.It is defined as strict refine if $\forall * a_{R(i)}, * a_{S(j) \varsubsetneqq} \subsetneq a_{R(i)}$, and the relation between $R$ and $S$ is denoted as $S>R$.

\section{2) Binary operator}

Definition 8: (Intersection Operator $\left.{ }^{\circledR}\right)$ Suppose temporal granular $* a_{R(i)}$ and temporal granular $* a_{R(i)}$ are derived from temporal relation $\mathrm{R}$ and $\mathrm{S}$ respectively, the intersection between $* a_{R(i)}$ and $* a_{S(j)}$ is equivalent to get a new granular which is composed of the ordered atomic time units in both $* a_{R(i)}$ and $* a_{S(j)}$.

Definition 9: (Union Operator (C) Suppose temporal granular $* a_{R(i)}$ and temporal granular $* a_{S(j)}$ is derived from temporal relation $R$ and $S$ respectively and is consecutive in time axis, the union between $* a_{R(i)}$ and $* a_{S(j)}$ is equivalent to get a new granular which is composed of ordered atomic time unit in either $* a_{R(i)}$ or $* a_{S(j)}$.

Definition 10: (Difference Operator $\Theta$ ) Suppose temporal granular $* a_{R(i)}$ and temporal granular $* a_{S(j)}$ is derived from temporal relation $R$ and $S$ respectively, the difference between $* a_{R(i)}$ and $* a_{S(j)}$ is equivalent to get a new granular which have the atomic time unit in $* a_{R(i)}$ except the atomic time unit contained in $* a_{R(i)}$ and $* a_{S(j)}$.

\section{3) Related properties}

Property 1: The number of temporal granular in temporal relation $R$ is larger than that in temporal relation $S$ if $S$ is the outcome of performing refine operator on $R$.

Property 2: The number of temporal granularl in temporal relation $R$ is smaller than that in temporal relation $S$ if $S$ is the outcome of performing coarse operator on $R$.

Property 3: Commutative law: $R \circledast S=S ® R ; R \Subset S=S \Subset R$

Associative law: $(R \AA S) \circledR Q=R \circledR(S \circledR Q)$;

$$
(R \odot S) \subseteq Q=R \odot(S \odot Q) ;
$$

Idempotent law: $R \circledast R=R ; R \Subset R=R$

Complement law: $R \Theta S=R \Theta(R \circledast S) ; S \Theta R=S \Theta(R \circledast S)$

Absorption law: $R \Subset(S \circledast R)=R ; R \circledast(S \odot R)=R$

Property 4: Any partition-based temporal relation of a given time object $U_{t}$ can be obtained by performing limited operator of coarse and refine.

\section{B. Relationship among Temporal Granular}

It is obvious that different temporal granular in the identical temporal relation have the unique order relation since all the temporal relations are the partitions of the given time objects. However, the temporal granular in different temporal relation have far more complicated relations which should be discussed. Based on the definition of binary granular operator, the relationship among temporal granular $* a_{R(i)}:=[o(w), o(x)], * a_{S(j)}:=[o(y), o(z)]$ in different temporal relation $\mathrm{R}$ and $\mathrm{S}$ are given as follows:

1) $* a_{R(i)}$ and $* a_{S(j)}$ have the relation of strict order if $* a_{R(i)}{ }^{\circledR} * a_{S(j)}=\varnothing$;

2) $* a_{R(i)}$ and $* a_{S(j)}$ have the relation of comparable order if $* a_{R(i)}{ }^{\circledR} * a_{S(j)}=o(q), q=1,2, \ldots \ldots$,

3) $* a_{R(i)}$ and $* a_{S(j)}$ have the relation of approximate order if $\forall o_{s \in}\left(* a_{R(i)^{-}} * a_{R(i)}\right) \circledR * a_{S(j)}, \quad \forall o_{t \in}\left(* a_{S(j)^{-}} * a_{R(i)}\right) \circledR * a_{S(j)}$ $\left(* a_{R(i)^{-}} * a_{R(i)}\right) \circledR * a_{S(j)} \neq \varnothing,\left(* a_{S(j)^{-}} * a_{R(i)}{ }^{\circledR} * a_{S(j)} \neq \varnothing\right.$ which meet $o_{s}<o_{t}(s<t)$, where the symbol $<$ denotes the atomic time unit $o_{s}$ occur before $o_{t}$;

$* a_{R(i)}$ and $* a_{S(j)}$ have the relation of weakened indiscernibility if $\left.\varnothing=\left(* a_{S(j)} \Theta * a_{R(i)}\right) \circledR \quad * a_{S(j)}\right\rfloor^{*} a_{R(i)} \Theta$ $\left.* a_{R(i)}\right){ }^{*} a_{S(j)}$ and $\forall o_{s \in}\left(* a_{R(i)} \Theta * a_{R(i)} \AA^{\circledR} * a_{S(j), \forall \mathrm{ot} \epsilon} * a_{S(j)}\right.$, which meet $\quad o_{s<} o_{t} \quad(s<t), \quad$ or $\varnothing \quad \varnothing \quad=\left(* a_{R(i)^{-}} \quad * a_{R(i)}\right) \mathbb{}$ $* a_{S(j) \subseteq}\left(* a_{S(j)} \Theta * a_{R(i)}\right) \circledR * a_{S(j)}$ and $\forall o_{t \in}\left(* a_{S(j)} \Theta * a_{R(i)}\right) \circledR * a_{S(j)}$, $\forall$ os $\epsilon^{*} a_{R(i)}$, which meet $o_{s} o_{t}(s<t)$. It can also be Viewed as inclusion relation in terms of set; Relation a) d) are shown in Fig. 4, where the symbol $\{\ldots o(q) \ldots\}$ denotes a series of consecutive atomic time unit while $o(q)$ denotes a certain atomic time unit.

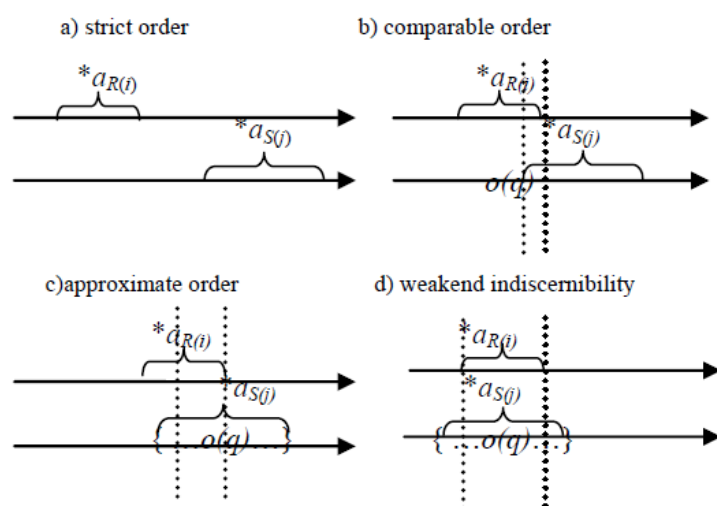

Fig. 4. Potential relation among different temporal granular.

\section{CONCLUSIONS}

Time series data mining (TSDM) is an active topic in recent years and has aroused many interests by scholars. By elaborating the construction of temporal relation and definition of the operator, a novel information system called TIS is developed. Compared with traditional granular theory on information system, new mechanism on granular transition is provided, which can be viewed as an extension of granular computing. The use of granular computing makes the structure of time series more hierarchical.

In the future the analysis of TIS needs to be promoted so that it is more suitable for algorithm design. Additionally, it is urgent to show the principle of optimal temporal granular and how to derive it with acceptable computation cost. The TIS also need to be implemented in real application to prove the robustness in time series with special properties such as complicated and uncertain.

\section{REFERENCES}

[1] L. Zhang and B. Zhang, Theory and Applications of Problem SolvingThe Quotient Space Granular Computation Theory and Applications, Beijing: Tsinghua University Press, 2007.

[2] Q. Liu, Rough Sets and Rough Reasoning, Academic Pubnlic, Beijing, 2001.

[3] R. H. Shumway and D. S. Stoffer, Time Series Analysis and its Applications: with R Example, Springer, 2010.

[4] Q. Liu and Q. Liu, "Granules and applications of granular computing in logical reasoning," Journal of Computer Research and Development, vol. 41, no. 4, pp. 546-551, 2004.

[5] A. R. Hmouz, W. Pedrycz, A. Balamash et al., "Description and classification of granular time series," Soft Computing, pp. 1-15, 2014.

[6] H. Yuwen, X. Jiucheng, and Z. Qianqian, "Research on evolution model of granular decision based on multiple time granularity," Computer Engineering and Applications, vol. 47, no. 20, pp. 117-120, 2011. 
[7] W. Pedrycz, W. Lu, X. Liu et al., "Human-centric analysis and interpretation of time series: a perspective of granular computing," Soft Computing, pp. 1-15, 2014.

[8] A. R. Hmouz, W. Pedrycz, A. Balamash et al., "Granular representation schemes of time series: A study in an optimal allocation of information granularity," in Proc. the 2013 IEEE Symposium on Foundations of Computational Intelligence (FOCI), 2013, pp. 44-51.

[9] X. Wang, K. Smith, and R. Hyndman, "Characteristic-based clustering for time series data," Data Mining and Knowledge Discovery, vol. 13, no. 3 , pp. 335-364, 2006

[10] H. Yuwen, X. Jiucheng, and Z. Qianqian, "Research on decision stability of evolution model of granular decision," Computer Science, vol. 12, 2012.

[11] Y. Y. Zuo, Y. Tang, Z. M. Shu et al., "Research on the characterization and the calculus of temporal granularities," Computer Science, vol. 37 , no. 12, pp. 114-119, 2010.

[12] Y. Y. Zuo, W. J. Tang, Y. Tang et al., "Vectorizing process and operation of the temporal spans," Computer Science, vol. 38, no. 11, pp. $171-175,2012$.

[13] W. Lu, W. Pedrycz, X. Liu et al., "The modeling of time series based on fuzzy information granules," Expert Systems with Applications, vol. 41 no. 8, pp. 3799-3808, 2014

[14] D. G. Li, D. Q. Miao, and H. Y. Zhang, "The theory models and approaches of granular computing," Journal of Fudan University, 2004, vol. 43 , no. 5 , pp. 837-841.

[15] D. G. Li, D. Q. Miao, and D. X. Zhang et al., "An overview of granular computing," Computer Science, vol. 32, no. 9, pp. 1-12, 2005.

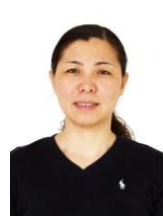

Min Tu was born in 1967. She received the B.E. in computer science from Jiangxi University in 1989. Now she is a professor at Jiangxi Police Institute. Her research interests include network communication and information security, etc.

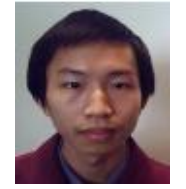

Yuanjian Zhang was born in 1990. He received B.E. from Software College of Nanchang University in 2012. Currently, he is a master candidate at Software College of Nanchang University. His research interests include granular computing, machine learning, etc. He is a student member of $\mathrm{CCF}$.

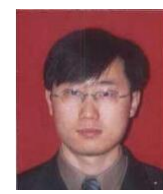

Jianfeng Xu was born in 1973. He received B.E, from electronic technology application from Nanchang University in 1996 and M.S. from computer technology application Nanchang University respectively in 2006 Now he is an associate professor at Software College of Nanchang University. His research interests include granular computing, machine learning, rough set etc. He is a member of IEEE and CCF.

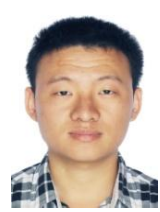

Yu Li was born in 1988. He received B.E. from Software College of Nanchang University in 2012. Currently, he is studying for a master's degree at Software College of Nanchang University. Now his research interest includes data mining, artificial intelligence and machine learning. 